\title{
Direct evidence of the multidimensionality of the free-energy landscapes of proteins revealed by mechanical probes
}

\author{
$\mathrm{Zu}$ Thur Yew \\ Institute of Molecular and Cell Biology, University of Leeds, Leeds LS2 9JT, United Kingdom \\ Michael Schlierf \\ Loomis Laboratory, University of Illinois at Urbana-Champaign, Urbana, Illinois 61801, USA \\ Matthias Rief* \\ Center for Integrated Protein Science Munich (CIPSM), Physik Department E22, TU München, \\ James-Franck Straße, 85748 Garching, Germany
}

Emanuele Paci $^{\dagger}$

Institute of Molecular and Cell Biology and School of Physics and Astronomy, University of Leeds, Leeds LS2 9JT, United Kingdom

(Received 11 August 2009; revised manuscript received 9 March 2010; published 30 March 2010)

\begin{abstract}
The study of mechanical unfolding, through the combined efforts of atomic force microscopy and simulation, is yielding fresh insights into the free-energy landscapes of proteins. Thus far, experiments have been mostly analyzed with one-dimensional models of the free-energy landscape. We show that as the two ends of a protein, filamin, are pulled apart at a speed tending to zero, the measured mechanical strength plateaus at $\sim 30 \mathrm{pN}$ instead of going toward zero, deviating from the Bell model. The deviation can only be explained by a switch between parallel pathways. Insightful analysis of mechanical unfolding kinetics needs to account for the multidimensionality of the free-energy landscapes of proteins, which are crucial for understanding the behavior of proteins under the small forces experienced in vivo.
\end{abstract}

DOI: 10.1103/PhysRevE.81.031923

PACS number(s): 87.10.-e

Single-molecule techniques such as atomic force microscopy and optical tweezers have been extensively used to probe the mechanical resistance of proteins by measuring the unfolding force or equivalently, the unfolding time. Due to the increased resolution of modern instruments, single molecule force spectroscopy has emerged as a valuable technique to detect and characterize transient states [1-3]. When coupled with a quantitative analysis of the unfolding kinetics or unfolding force distributions, these techniques provide a new window into the free-energy landscapes of proteins. The mechanical unfolding of a variety of proteins has now been studied. It is generally accepted that the mechanical unfolding of most single-domain proteins is a two-state process that can be described by the phenomenological Bell model [4]. Bell's model postulates that the average unfolding time decreases exponentially with the applied force: $\tau_{u}^{\text {mech }}(F)$ $=\tau_{u}^{m e c h}(0) \exp \left(-F x_{u}\right)$. The "naked" barrier height can then be approximated as $\ln \left[\tau_{u}^{\text {mech }}(0)\right]$, and the distance between the native state and the transition state along the pulling direction is given by the parameter $x_{u}$, which is assumed to be independent of the force. In constant velocity experiments, it is more convenient to analyze the unfolding forces, and an analogous expression links the unfolding force to the parameters $x_{u}$ and $\tau_{u}^{\text {mech }}(0)$. An improvement to the Bell model was recently proposed $[5,6]$ where the force dependence of $x_{u}$ was analytically evaluated for two specific one-dimensional (1D) energy profiles (i.e., the cusp and linear-cubic profiles).

\footnotetext{
*mrief@ph.tum.de

†e.paci@leeds.ac.uk
}

While such a treatment is undoubtedly more accurate [7], deviations from the Bell model are a second-order effect that can be masked by experimental error [8]. Another widely held view is that mechanical unfolding pathways are likely to be distinct from those traversed in denaturant induced unfolding. This stems from observations that the unfolding time in the limit of zero force, $\tau_{u}^{m e c h}(0)$, is different from the unfolding time determined in the limit of zero chemical denaturant [9]. The conclusions above are based on the analysis of mechanical unfolding kinetics in a limited range of forces or pulling speeds, which are usually quite large. More crucially, these conclusions are based on the assumption that the free-energy landscape of a protein can be approximated by a $1 \mathrm{D}$ profile. A consequence of such a view is that the possibility of both the mechanical and denaturant unfolding pathways coexisting at low forces is often neglected. Experiments on protein-ligand complexes performed in a broad range of pulling velocities have shown that the unfolding kinetics at low velocities can show strong deviations from the Bell model. In this regard, two classes of "anomalous" behavior have been reported in the literature. First, is the observation of two linear regimes in the plot of $F$ vs $\ln v$, indicating the presence of two sequential barriers [10]. Second, is the so-called "catch-slip" behavior where the mechanical resistance of the system initially increases with force before decreasing again in accordance with Bell's model [11-13]. Such behaviors have not been directly observed in single-domain proteins, which are often perceived as simple two-state systems. Simulation studies [14-16], however, have indicated that such anomalous behaviors can in principle be observed experimentally at low pulling velocities or forces. 
Here, we report the mechanical unfolding kinetics of dictyostelium discoideum filamin domain 4 (ddFLN4) in a broad range of pulling velocities and observed, in a singledomain protein, a strong deviation from the Bell model at very low pulling velocities. These strong deviations at pulling velocities of $1-20 \mathrm{~nm} / \mathrm{s}$ cannot be adequately explained by diffusion on $1 \mathrm{D}$ energy profiles. To explain the data, we present an alternative kinetic model involving two parallel unfolding pathways.

Single-molecule mechanical unfolding experiments were performed with a custom-built low-drift atomic force microscope (AFM). As probes we used gold-coated cantilevers (Biolever Type B, Olympus, Japan), which were calibrated using the equipartition theorem with typical spring constants $k_{c} \sim 6 \mathrm{pN} / \mathrm{nm}$. All experiments were carried out at room temperature in a freshly prepared PBS buffer $(10 \mathrm{mM} \mathrm{Na}-$ phosphate, $137 \mathrm{mM} \mathrm{NaCl}, 2.7 \mathrm{mM} \mathrm{KCl}, \mathrm{pH} 7.4)$. Typically $5 \mu \mathrm{l}$ of $1 \mathrm{mg} / \mathrm{ml}$ protein solution was added to $10 \mu \mathrm{l}$ of PBS on a freshly activated Ni-NTA surface and incubated for 5 min before starting the experiment [17]. The instrument was thermally equilibrated for approximately $30 \mathrm{~min}$ before taking data at pulling velocities of 1 and $20 \mathrm{~nm} / \mathrm{s}$ with a sampling rate of $20 \mathrm{kHz}$. The data were box filtered prior to analysis, and the unfolding forces were determined [18]. Recent improvements in the experimental setup, e.g., a modified fluid-cell design with a reduced light path through the solution, enabled us reduce to drift and acquire data at low pulling velocities. Furthermore, only the segment of a force extension trace, where ddFLN4 unfolding was expected, was recorded with a reduced pulling velocity. The force drift was estimated to be on the order of $0.04 \mathrm{pN} / \mathrm{s}$. The ddFLN1-5 construct, containing five immunoglobulin rod domains, was expressed and purified as described before [19]. ddFLN4 was unambiguously identified through its characteristic threestate unfolding mechanism exhibiting an obligatory unfolding intermediate. The experimental setup is depicted schematically in Fig. 1(a).

In this study, the previous [8] pulling velocity range (200$4000 \mathrm{~nm} / \mathrm{s}$ ) was expanded by $>200$ times to pulling velocities of $1 \mathrm{~nm} / \mathrm{s}$ and $20 \mathrm{~nm} / \mathrm{s}$ [Figs. 1(b) and 2(a)]. Consistent with the previous data, ddFLN4 unfolded at the lower pulling velocities through the characteristic unfolding intermediate [Fig. 1(b)], indicating that there is no significant change in the unfolding mechanism. In Fig. 2(a), the average unfolding force, $\langle F\rangle$, of the $\mathrm{N} \rightarrow \mathrm{I}$ transition is plotted against the pulling velocity. We now find a strong deviation from the Bell model; the velocity dependence of $\langle F\rangle$ around 1-20 $\mathrm{nm} / \mathrm{s}$ is distinct from that characterized around 200-4000 $\mathrm{nm} / \mathrm{s}$ (Fig. 2(a), black circles). Neither the Kramers approach [Fig. 2(a), blue line] described previously [8] nor an analytical treatment [5] for the 1D linear-cubic energy profile could reproduce the strong curvature observed for ddFLN4 at low pulling velocities [Fig. 2(a), green line].

The simplest explanation of the "anomalous" behavior at low pulling velocities is that there are two sequential barriers along the unfolding pathway of ddFLN4. The sequential barriers (SBs) model was first proposed for receptor-ligand unbinding by Evans and co-workers [10]. However, Fig. 2(b) shows that the SB model is incompatible with our data.

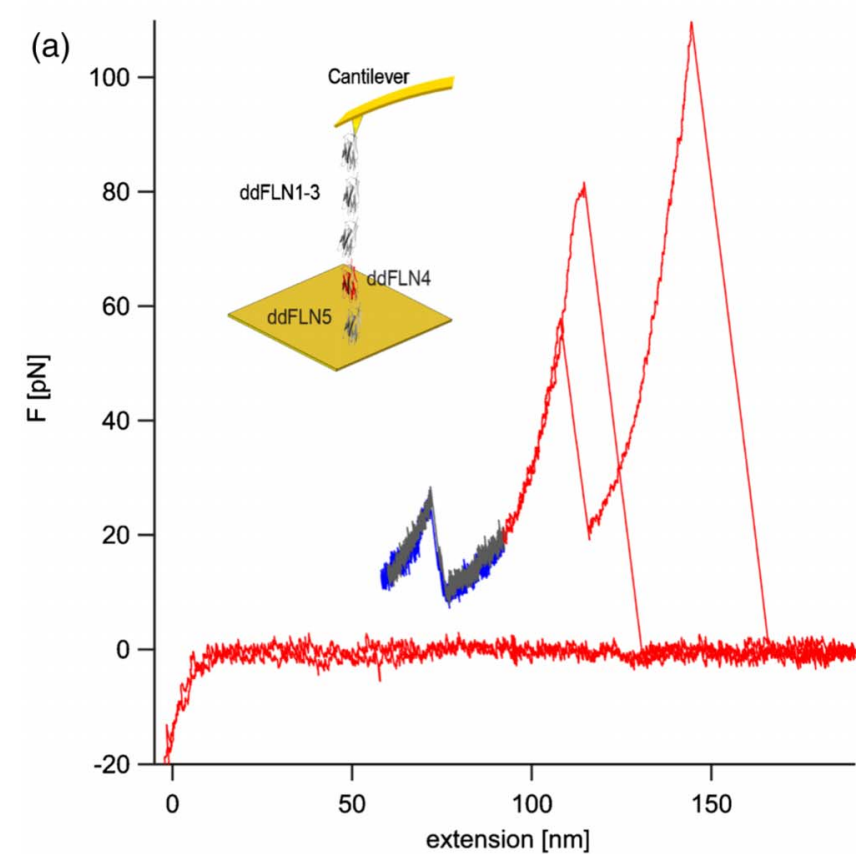

(b)
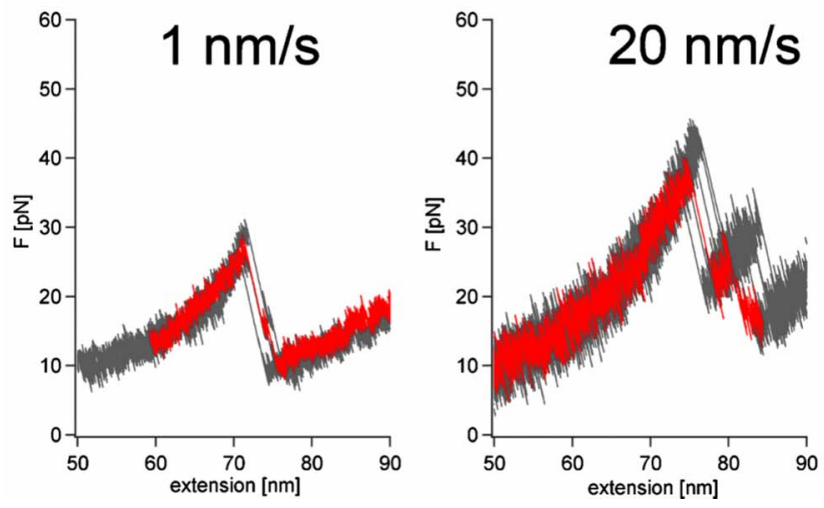

FIG. 1. (Color online) (a) Two segmented force extension traces of typical $1 \mathrm{~nm} / \mathrm{s}$ data. ddFLN1-5 was manually unfolded, the polypeptide chain was relaxed and then a 40-nm-long extension at 1 $\mathrm{nm} / \mathrm{s}$ to unfold ddFLN4 (blue and gray) followed by a $100 \mathrm{~nm} / \mathrm{s}$ segment (red) was performed. In the $100 \mathrm{~nm} / \mathrm{s}$ segment the extension was increased until ddFLN1-5 unfolds completely and detaches from the cantilever or the surface. Afterwards, the extension was reduced to zero in order to find the cantilever distance from the surface. One trace shows after a short force rise the detachment from cantilever or surface, the other trace shows unfolding of another ddFLN domain prior to increase and detachment at a higher extension. These traces show how closely the contour lengths of two different molecules for ddFLN4 unfolding match. Inset: sketch of the experimental setup with a ddFLN1-5 molecule attached between surface and cantilever. (b) Superimposition of typical force extension traces of ddFLN4 unfolding at a speed of $1 \mathrm{~nm} / \mathrm{s}$ and 20 $\mathrm{nm} / \mathrm{s}$. ddFLN4 can be identified unambiguously by the characteristic obligatory unfolding intermediate state.

The force distributions at 1 and $20 \mathrm{~nm} / \mathrm{s}$ cannot be both described by a single, unique set of $x_{u}$ and $\tau_{u}^{\text {mech }}(0)$ as required by the SB model [Fig. 2(b), green line]. Furthermore, the SB model is incompatible with the geometric constraints 

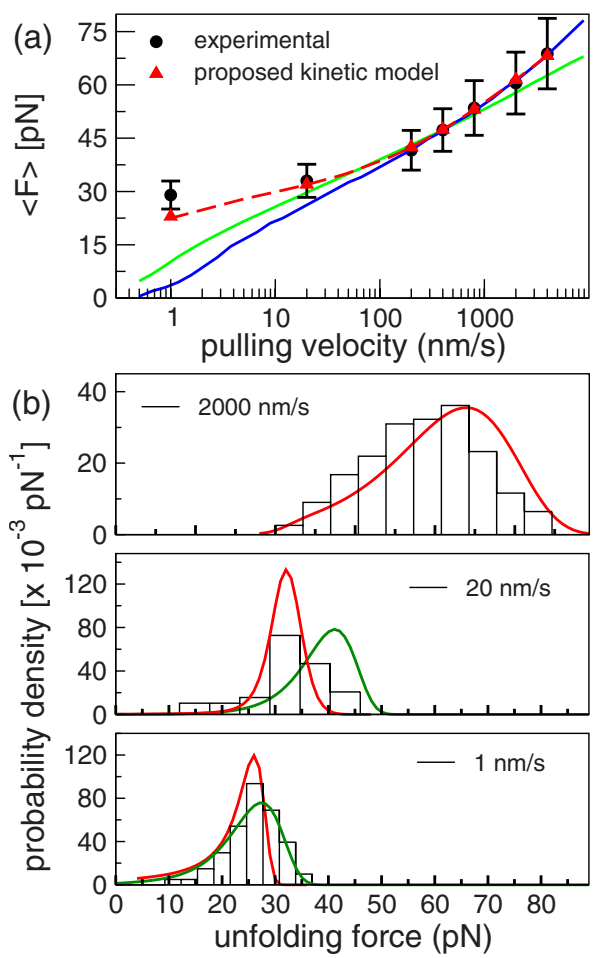

(c)

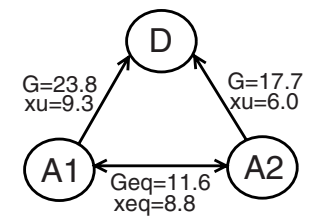

FIG. 2. (Color online) (a) The average unfolding force, $\langle F\rangle$, as function of the pulling velocity (black circles). Data points between 200 and $4000 \mathrm{~nm} / \mathrm{s}$ were previously published [8]. The bars on each point reflect the width of the unfolding force distribution. The blue line was obtained by computing the Kramers' rate as described in [8]. The green line was obtained using the linear-cubic model of Dudko et al. [5] $\left(\tau_{u}^{\text {mech }}(0)=213 \mathrm{~s}, x_{u}=11 \AA\right.$ and $\Delta G^{\ddagger}(0)$ $\left.=14.7 k_{B} T\right)$. The red triangles are the $\langle F\rangle$ computed from the kinetic scheme shown in part C. (b) Fits (red lines) of the experimental unfolding force histograms (black stairs) to the kinetic scheme shown in part C. The green line at $1 \mathrm{~nm} / \mathrm{s}$ was obtained by fitting the force distribution using the linear-cubic model of Dudko et al. $\left(\tau_{u}^{\text {mech }}(0)=7246 \mathrm{~s}, x_{u}=12 \AA\right.$ and $\Delta G^{\ddagger}(0)=25 k_{B} T$.) The green line at $20 \mathrm{~nm} / \mathrm{s}$ was computed based on the parameters determined at 1 $\mathrm{nm} / \mathrm{s}$, but with the pulling velocity $(20 \mathrm{~nm} / \mathrm{s})$ and the spacer length $(98 \mathrm{~nm})$ altered accordingly. (c) The proposed kinetic scheme. $G$ and $G_{e q}$ are in units of $k_{B} T$ and represent the activation and equilibrium free energy, respectively. The parameters $x_{u}$ and $x_{e q}$ are in units of $\AA$ and represent the unperturbed distance to the transition state, and between states A1 and A2, respectively.

imposed by the AFM setup. The two barriers in the SB model must at the same time be subjected to the external force. By increasing the force slowly, the outer barrier initially dominates the unfolding interactions, while at higher forces the inner barrier affects the forced unfolding behavior. This scenario for a single protein domain would imply that prior to unfolding via the outer barrier, several amino acids of the protein must have already unfolded via the inner barrier. However, a partial unfolding of several amino acids also changes the direction of force application as well as the mechanical unfolding reaction coordinate immediately [20]. The entire unfolding energy landscape and any previous force application to the previous barriers are thus reset. Such a scenario might be the case in forced receptor-ligand unbinding, where a large binding pocket holds a ligand with several distinct interactions.

The next simplest model is a kinetic scheme with two parallel unfolding pathways as shown in Fig. 2(c). To fit the unfolding force distributions to the proposed kinetic scheme, we (1) described each of the parallel unfolding pathways, and the equilibrium between states $\mathrm{A} 1$ and $\mathrm{A} 2$ using the kinetic model of Dudko et al. [5]; (2) calculated the loading rates [21] using a cantilever spring constant of $6 \mathrm{pN} / \mathrm{nm}$, and a wormlike chain representation [22] of the polymeric spacer with a persistence length of $0.5 \mathrm{~nm}$, and contour lengths of $79 \mathrm{~nm}(200-4000 \mathrm{~nm} / \mathrm{s})$ [8], $98 \mathrm{~nm}(20 \mathrm{~nm} / \mathrm{s})$, and $92 \mathrm{~nm}(1$ $\mathrm{nm} / \mathrm{s}$ ); (3) computed the theoretical unfolding force distributions according to the Evans-Ritchie formalism [23]; and (4) fitted the experimental unfolding force distributions from all seven pulling velocities simultaneously. We find that the proposed kinetic scheme is able to reproduce the force distributions and provide physically plausible parameters (Fig. 2). The proposed kinetic scheme fits the experimental data much better than 1D models [Fig. 2(a), blue and green lines, and Fig. 2(b), green line].

The slow pulling velocities (i.e., $<20 \mathrm{~nm} / \mathrm{s}$ ) allow the protein much more time to also sample unfolding pathways that occur at very low or zero force. Instead of the expected decrease in $\langle F\rangle$ at low pulling velocities toward zero, a significant deviation was observed. Such behavior deviates strongly from that predicted by any $1 \mathrm{D}$ model. The reasonable fits of the experimental data to the proposed kinetic scheme suggest that the curvature can be more generally explained by a switch between two parallel pathways. The proposed kinetic scheme is possibly the simplest model that is consistent with the AFM data, and can be viewed as a generalization of the SB model.

It was recently shown [24] for protein $L$ that Bell's model holds for forces up to $13 \mathrm{pN}$, in apparent contradiction with previous simulations that show the presence of anomalous curvatures for single-domain proteins [15]. Interestingly, the unfolding time extrapolated to zero force, $\tau_{u}^{\text {mech }}(0)$, is different from that measured using chemical denaturants [25]. Given that the unfolding rate is likely to be a smooth function of the applied force, the disparity between the independently determined unfolding times implies that there must exist a curvature that is distinct from those predicted by the Bell or more sophisticated 1D models. Indeed, the presence of a strong curvature such as the one observed for ddFLN4 hints at the existence of parallel pathways or a shift in the free-energy landscape regardless of the precise nature of the curvature (concave, convex or plateau).

More than just probing the mechanical strength of biomolecules, single molecule force spectroscopy has the potential to provide fundamental insights into the complex, multidimensional free-energy landscapes of biomolecules. We have shown that the measurement of force-induced unfolding kinetics can reveal signatures of the multidimensionality of 
the free-energy landscape of a single-domain protein. The results presented here reiterate the need to obtain kinetic data in a broad range of forces [26] and highlights the power of single molecule force spectroscopy to provide unique insights into the free-energy surfaces of proteins under physiologically realistic conditions.
E.P. and Z.T.Y. are supported by the Wellcome Trust and the University of Leeds. M.S. was supported in the framework of the Elite Network of Bavaria by the International Graduate School-NanoBioTechnology (IDK-NBT). We thank Michael Schleicher and Angelika A. Noegel for the ddFLN1-5 construct.
[1] I. Schwaiger, M. Schleicher, A. A. Noegel, and M. Rief, EMBO Rep. 6, 46 (2005).

[2] J. M. Fernandez and H. Li, Science 303, 1674 (2004).

[3] C. Cecconi, E. A. Shank, C. Bustamante, and S. Marqusee, Science 309, 2057 (2005).

[4] G. I. Bell, Science 200, 618 (1978).

[5] O. K. Dudko, G. Hummer, and A. Szabo, Phys. Rev. Lett. 96, 108101 (2006).

[6] G. Hummer and A. Szabo, Biophys. J. 85, 5 (2003).

[7] Z. T. Yew, S. Krivov, and E. Paci, J. Phys. Chem. B 112, 16902 (2008).

[8] M. Schlierf and M. Rief, Biophys. J. 90, L33 (2006).

[9] P. M. Williams, S. B. Fowler, R. B. Best, J. Toca-Herrera, K. A. Scott, A. Steward, and J. Clarke, Nature (London) 422, 446 (2003).

[10] R. Merkel, P. Nassoy, A. Leung, K. Ritchie, and E. Evans, Nature (London) 397, 50 (1999).

[11] E. Evans, A. Leung, V. Heinrich, and C. Zhu, Proc. Natl. Acad. Sci. U.S.A. 101, 11281 (2004).

[12] B. T. Marshall, M. Long, J. W. Piper, T. Yago, R. P. McEver, and C. Zhu, Nature (London) 423, 190 (2003).

[13] W. E. Thomas, E. Trintchina, M. Forero, V. Vogel, and E. V. Sokurenko, Cell 109, 913 (2002).

[14] R. B. Best, E. Paci, G. Hummer, and O. K. Dudko, J. Phys.
Chem. B 112, 5968 (2008).

[15] D. West, P. D. Olmsted, and E. Paci, J. Chem. Phys. 124, 154909 (2006).

[16] D. Bartolo, I. Derenyi, and A. Ajdari, Phys. Rev. E 65, 051910 (2002).

[17] M. Schlierf, F. Berkemeier, and M. Rief, Biophys. J. 93, 3989 (2007).

[18] M. Schlierf and M. Rief, J. Mol. Biol. 354, 497 (2005).

[19] I. Schwaiger, A. Kardinal, M. Schleicher, A. A. Noegel, and M. Rief, Nat. Struct. Mol. Biol. 11, 81 (2004).

[20] H. Dietz and M. Rief, Proc. Natl. Acad. Sci. U.S.A. 101, 16192 (2004).

[21] O. K. Dudko, G. Hummer, and A. Szabo, Proc. Natl. Acad. Sci. U.S.A. 105, 15755 (2008).

[22] J. F. Marko and E. D. Siggia, Macromolecules 28, 8759 (1995).

[23] E. Evans and K. Ritchie, Biophys. J. 72, 1541 (1997).

[24] R. Liu, S. Garcia-Manyes, A. Sarkar, C. L. Badilla, and J. M. Fernandez, Biophys. J. 96, 3810 (2009).

[25] D. J. Brockwell, G. S. Beddard, E. Paci, D. K. West, P. D. Olmsted, D. A. Smith, and S. E. Radford, Biophys. J. 89, 506 (2005).

[26] E. Evans, Annu. Rev. Biophys. Biomol. Struct. 30, 105 (2001). 